УДК 622.276 .63

\title{
ГРУППИРОВАНИЕ И ВЫДЕЛЕНИЕ ЗАЛЕЖЕЙ НЕФТИ В КАРБОНАТНЫХ КОЛЛЕКТОРАХ ПО ПРОДУКТИВНОСТИ НА СТАДИИ ПРОВЕДЕНИЯ ГЕОЛОГО-РАЗВЕДОЧНЫХ РАБОТ
}

\author{
Мухаметшин Вячеслав Шарифуллович 1 , \\ vsh@of.ugntu.ru
}

\author{
Кулешова Любовь Сергеевна 1 , \\ markl212@mail.ru
}

\author{
Сафиуллина Альбина Ринатовна' \\ safiullina.a.r@yandex.ru
}
1 Филиал Уфимского государственного нефтяного технического университета в г. Октябрьском, Россия, 452607, г. Октябрьский, ул. Девонская, 54а.

\begin{abstract}
Актуальность исследования обусловлена необходимостью оценки продуктивности месторождений Волго-Уральской нефтегазоносной провинции, сложенных карбонатным коллектором, на стадии составления проектной документации по разработке для определения рентабельности и стратегии эксплуатации объектов, в которых сосредоточены значительные по размерам запасы нефти, относящиеся к категории трудноизвлекаемых. Решение этой задачи позволяет расширить ресурсную базу региона и минимизировать риски при принятии управляющих решений.

Цель: провести группирование объектов в карбонатных коллекторах с созданием методики отнесения объектов, выходящих из разведки, к категории высоко- или низкопродуктивных с использованием косвенных данных.

Объекты: залежи нефрти в карбонатных коллекторах Волго-Уральской нефтегазоносной провинции.

Методы. При проведении группирования использован метод геолого-промыслового анализа, виды факторного анализа.

Результаты. Установлено, что граничным значением коэфффициента продуктивности залежей нефтии в карбонатных коллекторах, разделяющим месторождения на высоко- и низкопродуктивные, является значение, равное $10 \mathrm{~m} / \mathrm{cym} / \mathrm{MПа.} \mathrm{Показано,}$ что разделение залежей по продуктивности обусловливается емкостно-фрильтрационными и толщинными свойствами пород-коллекторов, а также условиями залегания пластов и свойствами пластовых фрлюидов. В среднем по высокопродуктивным объектам эти параметры больше. Низкопродуктивные объекты в среднем имеют большие значения вязкости и плотности пластовой нефти. Однако четко поставить в соответствие граничному значению коэффрициента продуктивности граничное значение какого-либо параметра невозможно, так как продуктивность залежей характеризуется комплексом значений параметров пласта и насыщающих его флюидов и лишь определенный набор этих значений позволяет оценить ее. Создана методика диффференциации объектов разработки в карбонатных коллекторах Волго-Уральской нефтеегазоносной провинции по коэфффициенту продуктивности на основе косвенных данных для решения задач выделения эксплуатационных объектов на многопластовых месторождениях; сравнения объектов при проведении анализа разработки и т. д.
\end{abstract}

\section{Ключевые слова:}

Коэфрфициент продуктивности, нефрть, карбонатные коллектора, эфффективность разработки, геолого-физические свойства пород.

\section{Введение}

Известно, что коэффициент продуктивности $\left(K_{\text {прод }}\right)$ в обобщенном виде учитывает фильтрационноемкостные свойства нефтяных пластов и во многом определяет степень извлечения нефти из недр, добывные возможности залежей, рентабельность разработки [1-12].

Значения продуктивности залежей используются при определении кондиционных значений емкостнофильтрационных свойств; дифференциации запасов; определении объектов эксплуатации; расчете показателей разработки; мониторинге процесса нефтеизвлечения (по изменению коэффициента продуктивности можно изучать эффективность условий вскрытия и заканчивания скважин, эффективность методов воздействия на призабойную зону пласта, изменение состояния призабойной зоны в процессе разработки); выборе мероприятий по регулированию и совершенствованию процесса добычи нефти; определении важнейшего показателя фильтрационных способностей пласта - коэффициента проницаемости, который широко используется при решении самых различных задач анализа и проектировании разработки залежей; расчете показателей разработки; определении режима работы скважин и т. д. [12-20]. Иначе говоря, дифференциация залежей по продуктивности с точки зрения выбора рациональной системы разработки имеет важное значение. Причем необходимо знать продуктивность залежей на стадии выхода месторождений из разведки. Однако эта стадия связана со слабой разбуренностью месторождений и, как следствие, с ограниченным количеством проведенных исследований скважин по гидродинамике, а также влиянием многочисленных «шумов», искажающих реальные свойства призабойной зоны в точке вскрытия ее скважиной из-за: загрязнения промывочной жидкостью пласта; образования высоковязких эмульсий; кольматации призабойной зоны пласта (ПЗП) в процессе вскрытия пласта бурением. Восстановление первоначальных свойств пласта происходит крайне медленно.

В этих условиях важным является устранение искажений реальной продуктивности через изучение 
влияния различных геолого-технологических параметров на изменение добывных возможностей скважин. Причем параметры должны достаточно надежно определяться на стадии проведения испытаний скважин на приток [21-24].

\section{Методы и материаль}

В работах $[25,26]$ предложено разделение залежей нефти в карбонатах Волго-Уральской нефтегазоносной провинции (ВУНГП) по эффективности разработки на средне- и низкопродуктивные. Граничным значением коэффициента продуктивности предложено считать величину, равную 20 т/сут/МПа. В этих работах делается вывод, что при $K_{\text {прод }} \geq 20$ т/сут/МПа эффективность разработки залежей в карбонатных коллекторах сопоставима с эффективностью разработки высокопродуктивных залежей, сложенных терригенным коллектором.

В то же время анализ эффективности разработки многочисленных залежей, сложенных карбонатным

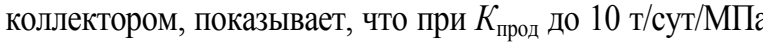
залежи нефти характеризуются весьма высокими показателями разработки, а процесс вытеснения в них идет аналогично вытеснению в терригенных коллекторах. И лишь при средних значениях продуктивности менее этой величины показатели разработки характеризуются весьма низкими значениями. Разработка этих объектов ведется на естественных режимах, а заводнение не приносит желаемых результатов. При этом конечная нефтеотдача к концу разработки этих объектов не превысит $15 \%$.

В связи с этим граничной продуктивностью, разделяющей высоко- и низкопродуктивные объекты в карбонатных коллекторах, должна быть принята продуктивность, равная 10 т/сут/МПа. Именно при этом значении залежи наиболее четко разделяются на высоко- и низкопродуктивные (рис. 2).

\section{Результаты и обсуждения}

Более чем по 400 залежам в карбонатных коллекторах ВУНГП были проведены расчёты на основе использования одной из разновидности метода факторного анализа - метода главных компонент (МГК) В качестве аргументов использовались параметры, определение которых с достаточной степенью точности проводится на стадии выхода месторождений из разведки. Значения параметров по выбранным объектам представлены в табл. 1.

Значения полученных первых четырех главных компонент имеют следующий вид:

$$
\begin{gathered}
\mathrm{Z}_{1}=-0,141 H_{\ni}+0,034 m_{\mathrm{r}}+0,032 m_{\mathrm{K}}+0,214 K_{\mathrm{H}}+ \\
\quad+0,002 \mu_{\mathrm{H}}+0,001 \mu_{\mathrm{o}}- \\
-0,025 \rho_{\mathrm{H}}+0,312 H_{\text {зал }}-0,056 P_{\text {нас }}-0,042 G+ \\
+0,321 P_{\text {пл }}+0,170 K_{\text {прон }}+0,333 t_{\text {пл }} ; \\
\mathrm{Z}_{2}=0,234 H_{\ni}-0,012 m_{\mathrm{r}}-0,001 m_{\mathrm{\kappa}}+0,020 K_{\mathrm{H}}+ \\
\quad+0,400 \mu_{\mathrm{H}}+0,400 \mu_{\mathrm{o}}+ \\
+0,109 \rho_{\text {н }}+0,007 H_{\text {зал }}-0,045 P_{\text {нас }}+0,039 G+ \\
+0,029 P_{\text {плл }}+0,315 K_{\text {прон }}+0,057 t_{\text {пл }} ;
\end{gathered}
$$

\begin{tabular}{|c|c|c|c|}
\hline \multirow{2}{*}{$\begin{array}{l}\text { Параметр } \\
\text { Parameter }\end{array}$} & \multicolumn{3}{|c|}{$\begin{array}{l}\text { Значения параметров } \\
\text { Parameter values }\end{array}$} \\
\hline & \begin{tabular}{|c|} 
мини- \\
мальноее \\
min
\end{tabular} & $\begin{array}{l}\text { среднее } \\
\text { average }\end{array}$ & $\begin{array}{c}\text { макси- } \\
\text { мальное } \\
\max \end{array}$ \\
\hline $\begin{array}{l}\text { Эффективная нефтенасыщенная } \\
\text { толщина } H_{3}, \text { м } \\
\text { Net oil pay } H_{3}, \mathrm{~m}\end{array}$ & 0,5 & 5,9 & 102,3 \\
\hline $\begin{array}{l}\text { Коэффициент пористости (по гео- } \\
\text { физике) } m_{\mathrm{r}}, \% \\
\text { Porosity factor (geophysics) } m_{\text {г }}, \%\end{array}$ & 3,0 & 13,3 & 24,2 \\
\hline $\begin{array}{l}\text { Коэффициент пористости (по кер- } \\
\text { ну) } m_{\kappa}, \% \\
\text { Porosity factor (by core) } m_{\kappa}, \%\end{array}$ & 2,0 & 13,2 & 28,0 \\
\hline $\begin{array}{l}\text { Коэффициент нефтенасыщенно- } \\
\text { сти, } K_{\text {н }} \\
\text { Oil saturation factor, } K_{\text {н }}\end{array}$ & 0,45 & 0,78 & 0,96 \\
\hline $\begin{array}{l}\text { Вязкость нефти } \mu_{\mathrm{H}}, \mathrm{MПа} \cdot \mathrm{c} \\
\text { Oil viscosity } \mu_{\mathrm{H}}, \mathrm{mPa} \cdot \mathrm{s}\end{array}$ & 0,9 & 23,0 & 390,0 \\
\hline $\begin{array}{l}\text { Относительная вязкость нефти } \mu_{\mathrm{o}} \\
\text { Specific oil viscosity } \mu_{\mathrm{o}}\end{array}$ & 0,7 & 16,3 & 229,0 \\
\hline $\begin{array}{l}\text { Плотность нефти } \rho_{\mathrm{H}}, \mathrm{\kappa г} / \mathrm{M}^{3} \\
\text { Oil density } \rho_{\mathrm{H}}, \mathrm{kg} / \mathrm{m}^{3}\end{array}$ & 720 & 862 & 958 \\
\hline $\begin{array}{l}\text { Глубина залегания } H_{\text {зал}}, \mathrm{M} \\
\text { Occurrence depth } H_{\text {зал }}, \mathrm{m}\end{array}$ & 230 & 1269 & 2570 \\
\hline $\begin{array}{l}\text { Давление насыщения } P_{\text {нас }}, \text { МПа } \\
\text { Saturation pressure } P_{\text {нас }}, \text { МР }\end{array}$ & 0,5 & 5,0 & 21,3 \\
\hline $\begin{array}{l}\text { Пластовый газовый фактор } G, \mathrm{~m}^{3} / \mathrm{T} \\
\text { Formation gas-oil ratio } G, \mathrm{~m}^{3} / \mathrm{t}\end{array}$ & 1,8 & 25,0 & 207,0 \\
\hline $\begin{array}{l}\text { Начальное пластовое давление } P_{\text {пл }} \text {, } \\
\text { МПа } \\
\text { Initial reservoir pressure } P_{\text {пл }}, \mathrm{MPa}\end{array}$ & 2,8 & 12,5 & 28,7 \\
\hline $\begin{array}{l}\text { Начальная пластовая температура } \\
t_{\text {пл }}, \mathrm{K} \\
\text { Initial reservoir temperature } t_{\text {пл, }}, \mathrm{K}\end{array}$ & 278 & 298 & 327 \\
\hline $\begin{array}{l}\text { Коэффициент проницаемости } \\
K_{\text {прон }}, 10^{-3} \text { мкм }^{2} \\
\text { Permeability coefficient } K_{\text {прон }}, 10^{-3} \\
\text { mkm }^{2}\end{array}$ & 6 & 85 & 1065 \\
\hline
\end{tabular}

$$
\begin{gathered}
\mathrm{Z}_{3}=0,491 H_{\ni}-0,047 m_{\mathrm{r}}-0,038 m_{\mathrm{K}}+0,027 K_{\mathrm{H}}+ \\
+0,084 \mu_{\mathrm{H}}+0,086 \mu_{\mathrm{o}}- \\
-0,208 \rho_{\mathrm{H}}-0,082 H_{\text {зал }}+0,287 P_{\text {нас }}+0,383 G- \\
\quad-0,066 P_{\text {пл }}+0,113 K_{\text {прон }}-0,079 t_{\text {плл }} ; \\
\mathrm{Z}_{4}=-0,102 H_{\ni}+0,439 m_{\mathrm{r}}+0,441 m_{\mathrm{K}}+0,251 K_{\mathrm{H}}- \\
0,035 \mu_{\mathrm{H}}-0,032 \mu_{\mathrm{o}}- \\
-0,116 \rho_{\mathrm{H}}-0,024 H_{\text {зал }}-0,087 P_{\text {нас }}+0,011 G+ \\
+0,003 P_{\text {пл }}+0,200 K_{\text {прон }}+0,038 t_{\text {плл }} .
\end{gathered}
$$

Таблица 1. Значения параметров объектов исследования

Table 1. Values of research objects parameters

После использования МГК в осях главных компонент (ГК) на основании установленной границы были выделены зоны приуроченности высоко- и низкопродуктивных объектов (табл. 2).

В зоне I все объекты являются высокопродуктивными, в зонах III и IV - лишь часть. Зона II включает низкопродуктивные объекты. При попадании объекта в зоны I и II можно утверждать однозначно о принадлежности его к высоко- или низкопродуктивной группе. Более четкое разделение объектов зон III и IV по продуктивности получено после проведения дискриминантного анализа (рис. 1). 

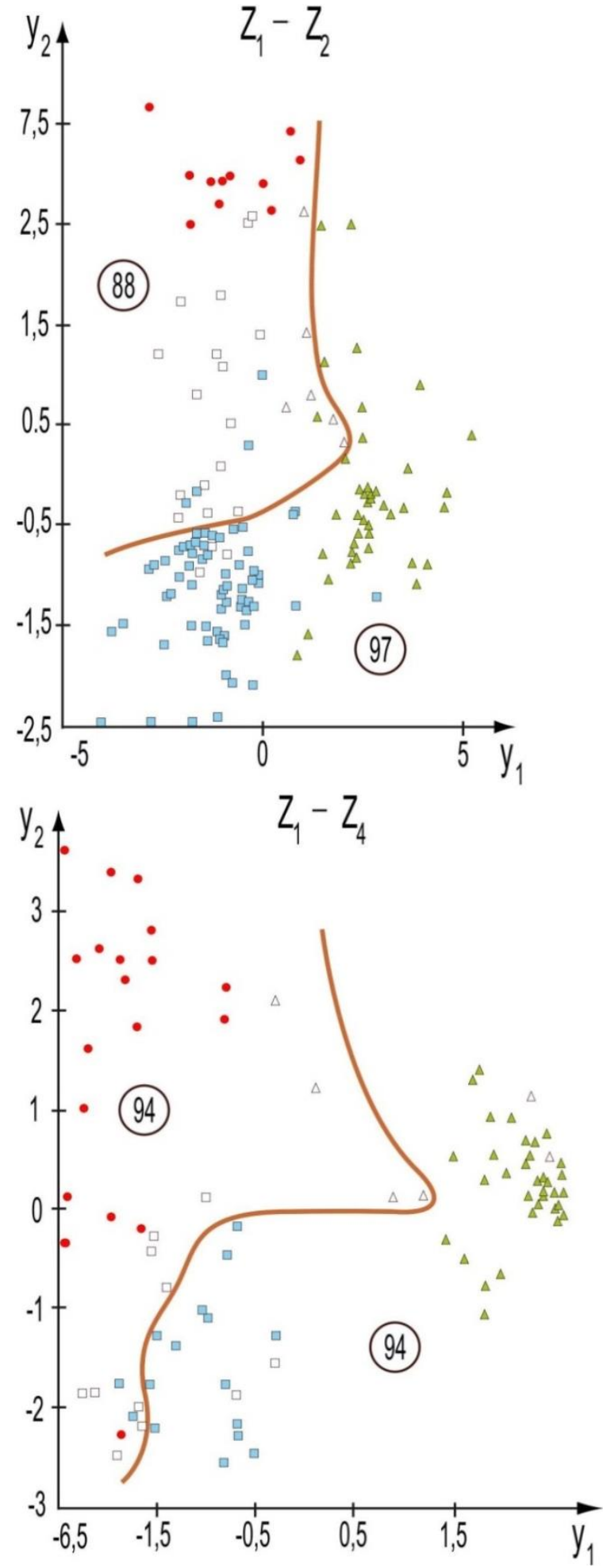

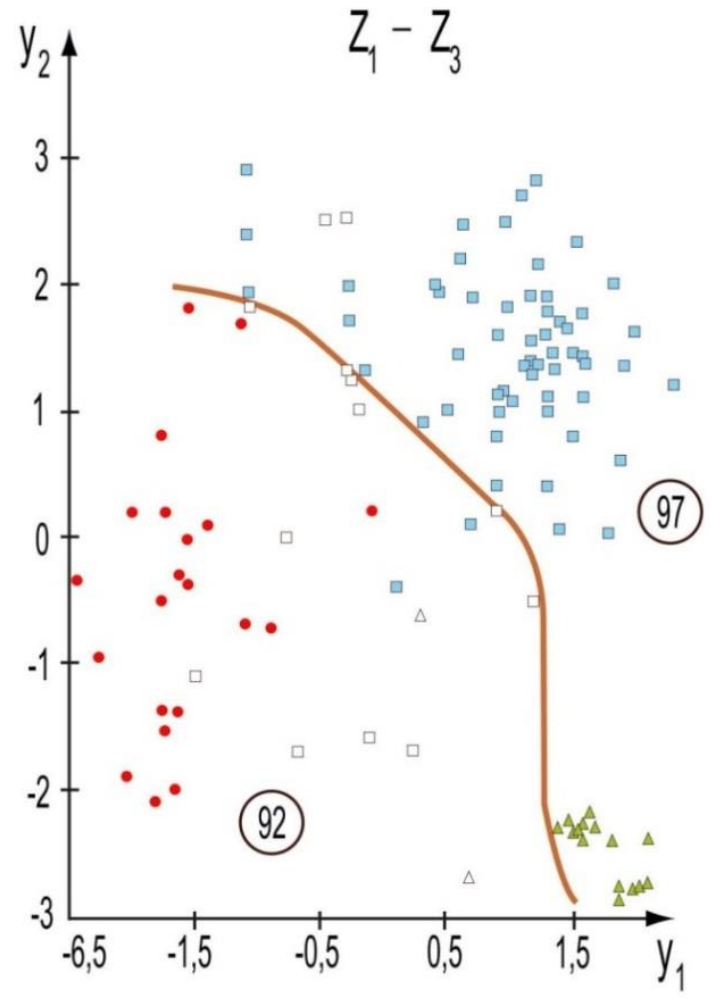

Рис. 1. Распределение высоко- $и$ низкопродуктивных объектов в осях канонических переменных $y_{1}-y_{2}$ : - объекты зоны І в осях главных компонент; $\square$, $\square-$ объекты зоны ІІІ в осях главных компонент; $\triangle, \triangle$ - объектыл зоны IV в осях главных компонент; ○ $\square, \triangle$ - высокопродуктивные объектьл; $\square, \triangle$ - низкопродуктивныле объекты; - граница высоко- и низкопродуктивных объектов; 97 - процент высоко- или низкопродуктивных объектов в выделенных областях; $Z_{l}$, $Z_{2}, Z_{3}, Z_{4}$ - главные компоненты

Fig. 1. Distribution of highly-and non-productive facilities in the axes of the canonical variables $y_{1}-y_{2}$ : facilities of zone $I$ in the axes of the principal components; $\square$, $\square$-facilities of zone III in the axes of the main components; $\triangle, \triangle$-facilities of zone IV in the axes of the main components; $\bigcirc, \square, \triangle-$ high productivity facilities; $\square, \triangle$ - low-productivity facilities; - the border of high- and lowproductivity facilities; (97) - percentage of high-or low productivity facilities in the selected areas; $Z_{1}$, $Z_{2}, Z_{3}, Z_{4}-$ main components

Таблица 2. Интервалы изменения значений главных компонент зон высоко- и низкопродуктивных объектов

Table 2. Changes in the values of the main components of the zones of high and low productivity objects

\begin{tabular}{|c|c|c|c|c|c|}
\hline \multirow{2}{*}{$\begin{array}{l}\text { Оси главных компонент } \\
\text { Principal component axes }\end{array}$} & \multirow{2}{*}{$\begin{array}{c}\text { Главные компоненты } \\
\text { Principal components }\end{array}$} & \multicolumn{4}{|c|}{$\begin{array}{c}\text { Значения интервалов изменения значений главных компонент по зонам } \\
\text { Values of the intervals of changes in the values of the main components by zones }\end{array}$} \\
\hline & & I & II & III & IV \\
\hline \multirow{2}{*}{$\mathrm{Z}_{1}-\mathrm{Z}_{2}$} & $\mathrm{Z}_{1}$ & $-2,0 \ldots 0,7$ & $0,7 \ldots 4,0$ & $0,7 \ldots 4,0$ & $-0,5 \ldots 0,1$ \\
\hline & $\mathrm{Z}_{2}$ & $-1,0 \ldots 4,0$ & $0,5 \ldots 1,5$ & $0,5 \ldots-1,0$ & $0,7 \ldots 0,4$ \\
\hline \multirow{2}{*}{$\mathrm{Z}_{1}-\mathrm{Z}_{3}$} & $\mathrm{Z}_{1}$ & $0,7 \ldots 4,0$ & $-2,0 \ldots 0,7$ & $0,7 \ldots 4,0$ & $-0,5 \ldots 0,1$ \\
\hline & $\mathrm{Z}_{3}$ & $0,7 \ldots 5,0$ & $-1,0 \ldots 5,0$ & $-0,5 \ldots 0,7$ & $-0,1 \ldots 0,5$ \\
\hline \multirow{2}{*}{$\mathrm{Z}_{1}-\mathrm{Z}_{4}$} & $\mathrm{Z}_{1}$ & $0,7 \ldots 4,0$ & $2,0 \ldots 0,7$ & $0,7 \ldots 4,0$ & $-0,5 \ldots 0,1$ \\
\hline & $\mathrm{Z}_{4}$ & $0,7 \ldots 2,0$ & $-3,0 \ldots 2,0$ & $0,7 \ldots-0,7$ & $-0,3 \ldots 0,5$ \\
\hline
\end{tabular}


В осях канонических переменных $\mathrm{y}_{1}-\mathrm{y}_{2}$ процент высоко- или низкопродуктивных объектов в выделенных областях составляет от 88 до $97 \%$, что является высоким показателем. Для сравнения аналогичная операция была проведена при граничных значениях $K_{\text {прод }}$ равных $6,8,12,14,16,18,20$ т/сут/МПа, откуда видно (рис. 2), что при значении $K_{\text {прод}}=10 \mathrm{~T} / \mathrm{cyт} / \mathrm{MПа} \mathrm{объекты} \mathrm{наиболее} \mathrm{четко} \mathrm{делятся}$ на высоко- и низкопродуктивные.

Анализ распределений, представленных на рис. 2, и уравнений канонических переменных (табл. 3) показывает, что разделение залежей по продуктивности обусловливается как емкостно-фильтрационными, так и толщинными свойствами пластов, а также свойствами пластовых флюидов и условиями залегания. В среднем по высокопродуктивным объектам эти параметры больше. Низкопродуктивные объекты в среднем имеют большие значения вязкости и плотности пластовой нефти. Однако четко поставить в соответствие граничному значению коэффициента продуктивности граничное значение какого-либо параметра, как видно из рис. 3 , не представляется возможным. Иначе говоря, продуктивность залежей характеризуется комплексом значений параметров пласта и насыщающих его флюидов и лишь определенный набор этих значений позволяет оценить ее.

Таблица 3. Уравнения канонических переменных $y_{1}-y_{2}$ для разделения залежей по продуктивности

Table 3. Equations of canonical variables $y_{1}-y_{2}$ for deposits separation by their productivity

\begin{tabular}{|c|c|c|}
\hline $\begin{array}{c}\text { Для зоны } \\
\text { III, IV в } \\
\text { координатах } \\
\text { For zone } \\
\text { III, IV in } \\
\text { coordinates }\end{array}$ & $\begin{array}{c}\text { Уравнение } \\
\text { Equation }\end{array}$ & $\begin{array}{c}\text { Номер } \\
\text { уравнения } \\
\text { Equation } \\
\text { number }\end{array}$ \\
\hline \multirow{2}{*}{$Z_{1}-Z_{2}$} & $\begin{array}{c}y_{1}=46,5+0,052 H_{3}+0,058 m_{\mathrm{r}}- \\
-7,05 K_{\mathrm{H}}+0,12 \mu_{\mathrm{o}}- \\
-0,004 \rho_{\mathrm{H}}-0,128 t_{\text {пл }}-0,002 K_{\text {прон }}\end{array}$ & (5) \\
\hline & $\begin{array}{c}y_{2}=1,25+0,072 H_{3}+0,217 m_{\mathrm{K}}- \\
-3,579 K_{\text {н }}-0,018 \mu_{\mathrm{o}}- \\
-0,007 t_{\text {пл }}+0,007 K_{\text {прон }} ;\end{array}$ & (6) \\
\hline \multirow{2}{*}{$Z_{1}-Z_{3}$} & $\begin{array}{c}y_{1}=-12,02-0,187 H_{3}+0,018 \rho_{\mathrm{н}}- \\
-0,0002 H_{\text {зал }}-0,021 G- \\
-0,004 K_{\text {прон }} ;\end{array}$ & (7) \\
\hline & $\begin{aligned} y_{2}= & -5,07-0,008 H_{9}-0,0009 \rho_{\mathrm{н}}+ \\
& +0,003 H_{\text {зал }}-0,01 G+ \\
& +0,002 K_{\text {прон }} ;\end{aligned}$ & (8) \\
\hline \multirow{2}{*}{$Z_{1}-Z_{4}$} & $\begin{aligned} y_{1}= & 29,78-0,01 H_{3}-0,178 m_{\mathrm{r}}+ \\
& +0,009 \rho_{\mathrm{н}}-0,002 H_{\text {зал }}- \\
& -0,102 t_{\text {пл }}-0,005 K_{\text {прон }} ;\end{aligned}$ & (9) \\
\hline & $\begin{aligned} y_{2}= & 27,31+0,057 H_{\ni}+0,345 m_{\mathrm{r}}- \\
& -0,009 \rho_{\mathrm{r}}-0,001 H_{\text {зал }}- \\
& -0,077 t_{\text {пл }}+0,0005 K_{\text {прон. }}\end{aligned}$ & (10) \\
\hline
\end{tabular}

Иначе говоря, при составлении проектных доку-

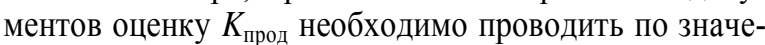
ниям параметров, входящих в уравнения ГК, и по расположению объектов в пространстве первых четырех главных компонент. В случае если объект попал в трех плоскостях в зоны I и II, можно однозначно утверждать о принадлежности его к высоко- или низкопродуктивной группам. В случае попадания объекта в зоны неопределенности (зоны III, IV) необ- ходимо рассчитать значения канонических переменных для него и определить его местоположение на плоскости (рис. 1).
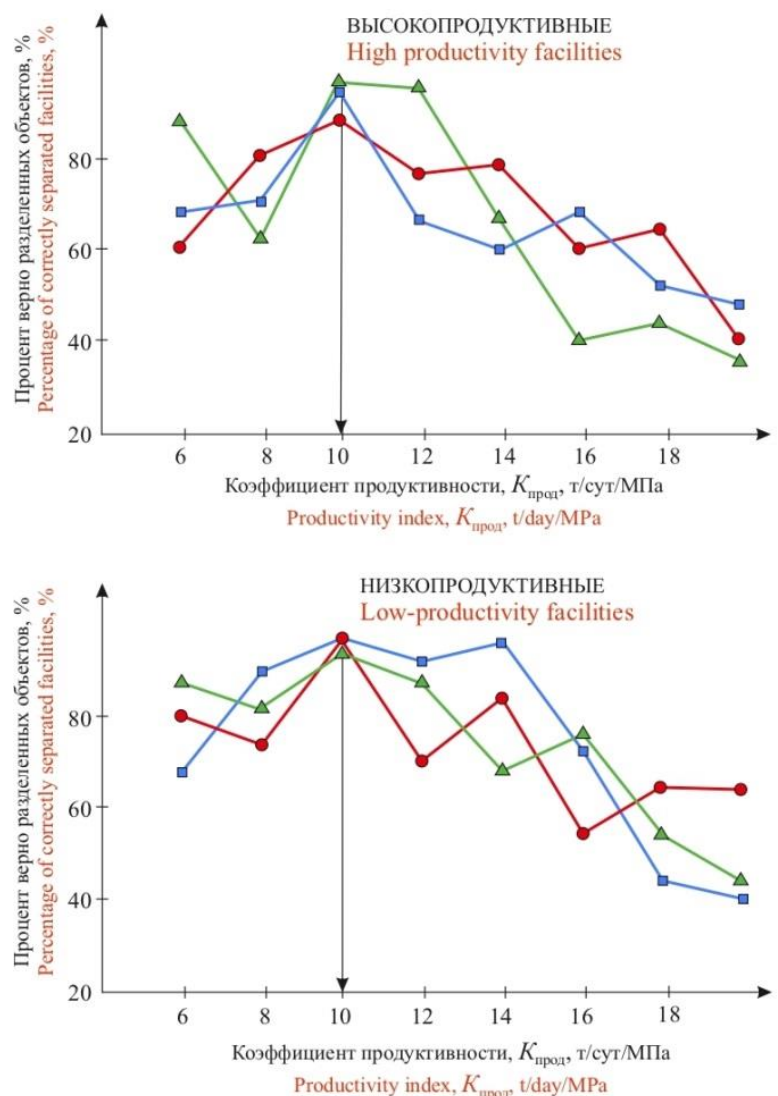

Pис. 2. Эффективность разделения высоко- и низкопродуктивных объектов при различных граничных значениях продуктивности: -0- - для залежей зон в осях $Z_{1}-Z_{2}$; - $\square-$ - дл залежей зон в осях $Z_{I}-Z_{3} ; \triangle-$ для залежей зон в осях $Z_{1}-Z_{4}$

Fig. 2. Efficiency of high- and low-productivity facilities separation at various boundary values of productivity: $-0-$ - for reservoirs of zones in the axes $Z_{1}-Z_{2}$; $\square-$ for reservoirs of zones in the axes $Z_{1}-Z_{3} ;-\triangle-$ for reservoirs of zones in the axes $Z_{1}-Z_{4}$

При оценке продуктивности залежей в стадии проведения геологоразведочных работ необходимо знать среднее значение коэффициента проницаемости, которое входит в уравнения (1)-(10). В этой стадии определение его возможно на основании лабораторных исследований керна или использования эталонных зависимостей проницаемости от пористости, построенных для залежей, близких по строению и коллекторским свойствам исследуемой.

Использование этих зависимостей возможно, если имеются значения пористости по керну, однако незначительная погрешность при определении средних значений пористости по керну и по данным геофизических исследований (рис. 4) позволяет оценивать проницаемость и в случае, если керн по залежи не был отобран. Проницаемость, определенная таким образом, будет довольно приближенной, однако для 
решения задачи качественного разделения объектов по продуктивности в условиях отсутствия более надежной информации ее использование будет вполне оправданным. Наличие тесной взаимосвязи
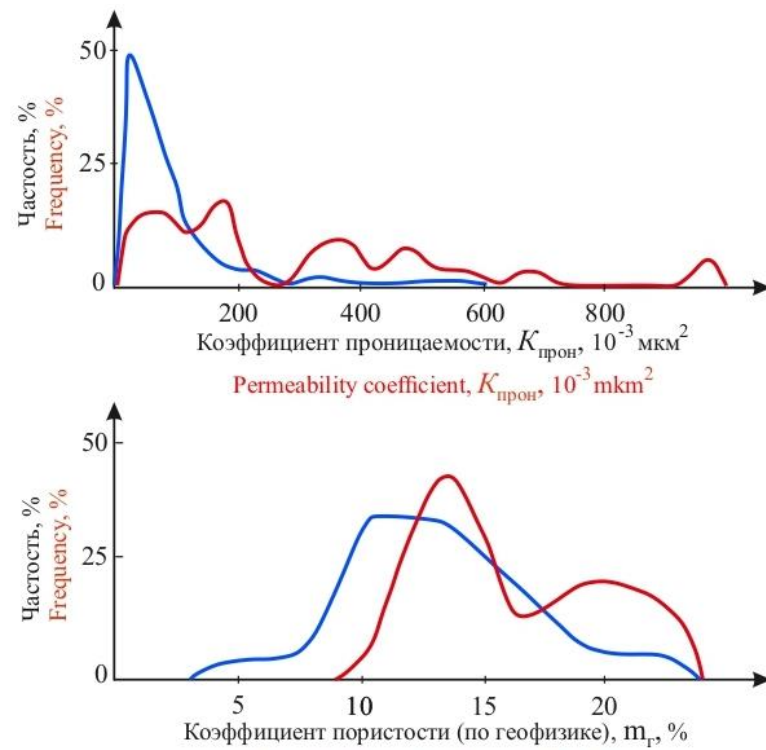

Porosity factor (geophysics), $\mathrm{m}_{\mathrm{r}}, \%$

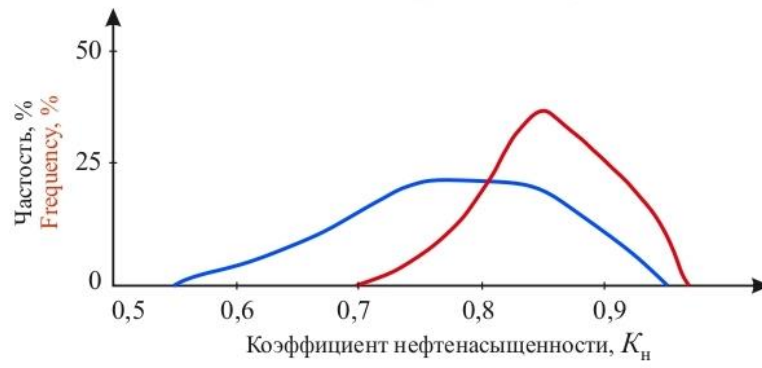

Oil saturation factor, $K_{\mathrm{H}}$
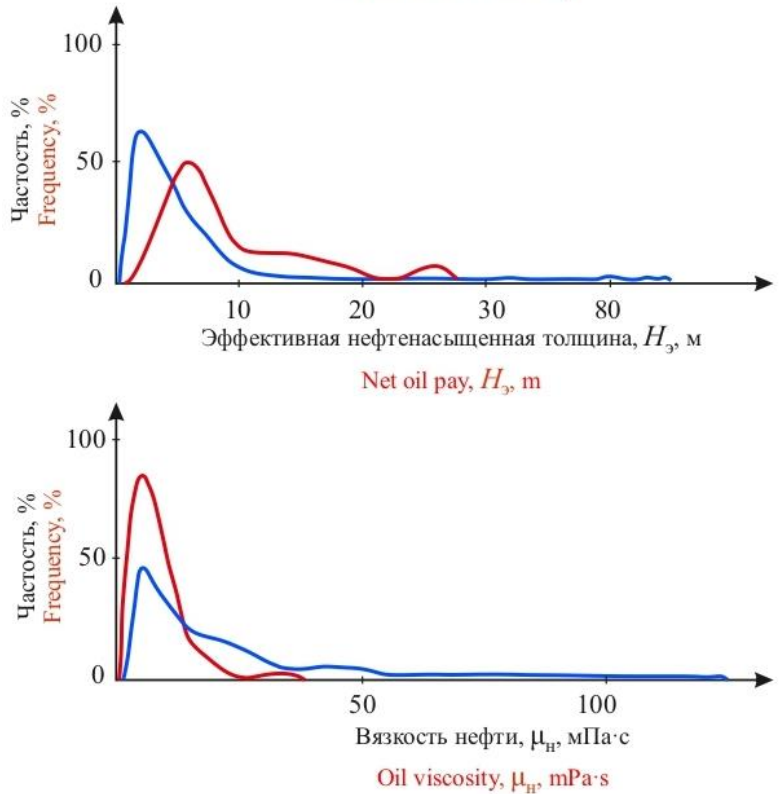

между параметрами $m_{\mathrm{\kappa}}$ и $m_{\Gamma}$ (рис. 4) также позволяет в формулах (1)-(10) заменить $m_{\mathrm{\kappa}}$ на $m_{\text {г }}$ в случае отсутствия керна и лабораторных исследований его пористости.

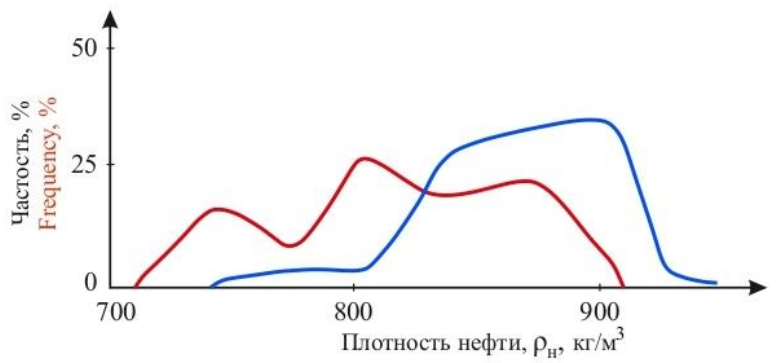

Oil density, $\rho_{\mathrm{H}}, \mathrm{kg} / \mathrm{m}^{3}$

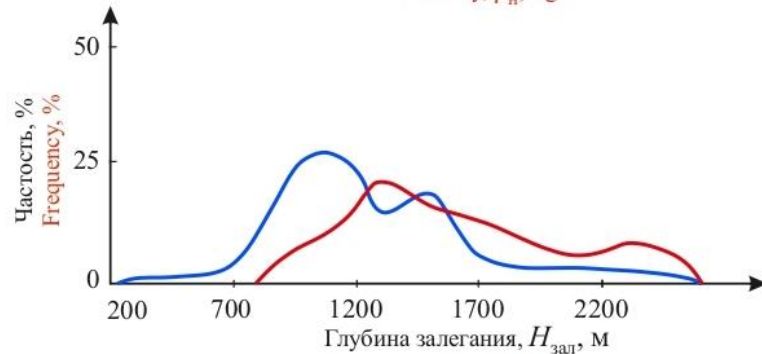

Occurrence depth, $H_{\text {зал, }}, \mathrm{m}$
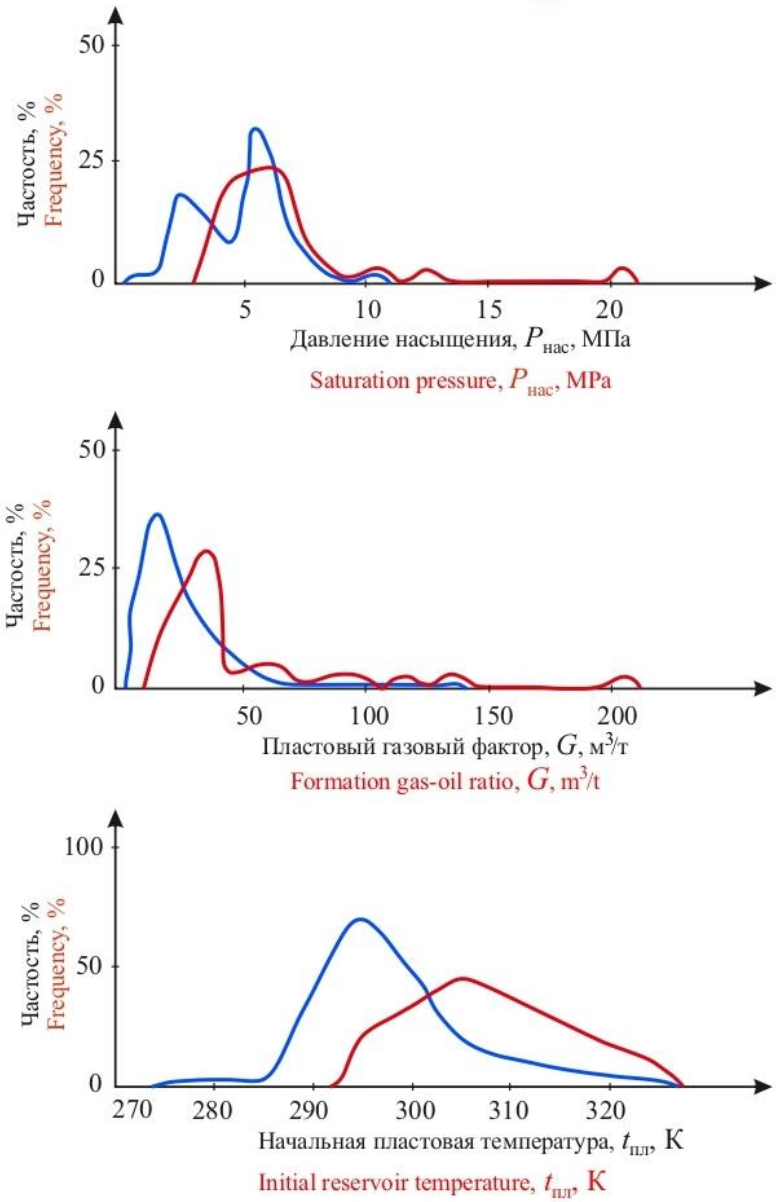

Рис. 3. Плотность распределения параметров высоко- и низкопродуктивных объектов: - кривая распределения низкопродуктивных объектов; - кривая распределения высокопродуктивных объектов

Fig. 3. Density of high- and low-productivity facilities parameters distribution: — — distribution curve of lowproductivity facilities; - distribution curve of high-productivity facilities 


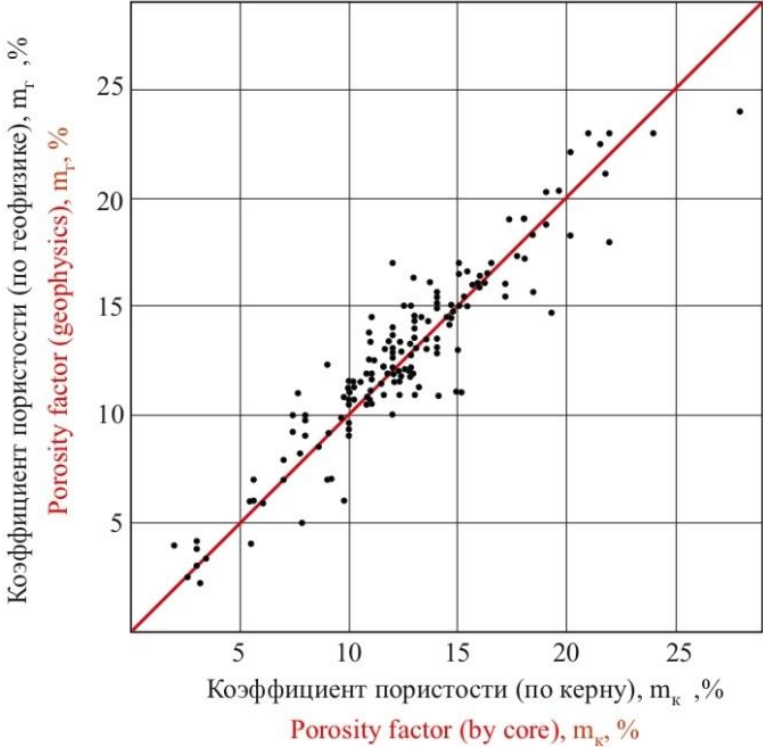

\section{Выводы}

1. Установлено, что граничным значением коэффициента продуктивности залежей нефти в карбонатных коллекторах, разделяющим месторождения на высоко- и низкопродуктивные, является значение, равное 10 т/сут/МПа.

2. Выявлены геолого-физические параметры продуктивных карбонатных пород, оказывающие

\section{СПИСОК ЛИТЕРАТУРЫ}

1. Муслимов Р.Х. Современные методы повышения нефтеизвлечения: проектирование, оптимизация и оценка эффективности. - Казань: ФЭН, 2005. - 688 с.

2. Economides J.M., Nolte K.I. Reservoir stimulation. - West Sussex England: John Wiley and Sons, 2000. - 856 p.

3. Мухаметшин В.В., Кулешова Л.С. Обоснование систем заводнения низкопродуктивных залежей нефти в условиях ограниченного объема информации // SOCAR Proceedings. - 2019. № 2. - C. 16-22. DOI: 10.5510/OGP20190200384.

4. Byrnes A.P., Bhattacharya S. Influence of initial and residual oil saturation and relative permeability on recovery from transition zone reservoirs in shallow-shelf carbonates // SPE/DOE Symposium on Improved Oil Recovery. - Tulsa, Oklahoma, USA, 22-26 April 2006. - 11 p. DOI: 10.2118/99736-MS

5. Мухаметшин В.В. Оценка эффективности использования нанотехнологий после завершения строительства скважин, направленных на ускорение ввода месторождений нефти в разработку // Нанотехнологии в строительстве. - 2018. T. 10. - № 1. - C. 113-131. DOI: 10.15828/2075-8545-2018-101-113-131.

6. Мухаметшин В.В. Повышение эффективности управления объектами добычи нефти с использованием метода аналогий // SOCAR Proceedings. - 2020. - № 4. - C. 42-50. DOI: 10.5510/OGP20200400464.

7. Back M.J., Kirk G. An integrated portfolio management approach for more effective business planning // SPE Hydrocarbon Economics and Evaluation Symposium. - Calgary, Alberta, Canada, 24-25 September 2012. - 12 p. DOI: 10.2118/162748-MS

8. Оптимизация выработки запасов из водонефтяных зон горизонта D3ps Шкаповского нефтяного месторождения с помощью горизонтальных скважин / Р.Ф. Якупов, В.Ш. Мухаметшин, И.Н. Хакимзянов, В.Е. Трофимов // Георесурсы. 2019. - T. 21. - № 3. - C. 55-61. DOI: 10.18599/grs.2019.3.55-61

9. Burchette T. Carbonate rocks and petroleum reservoirs: a geological perspective from the industry // Geological Society of London Special Publications. - 2012. - V. 370. - P. 17-37.

10. Roehl P.O., Choquette P.W. Carbonate petroleum reservoirs. New York: Springer-Verlag, 1985. - 622 p.
Pис. 4. Сопоставление значений определения пористости по керну $\left(m_{\kappa}\right)$ и по данным геофизических исследований $\left(m_{2}\right)$

Fig. 4. Comparison of the values of porosity determination on core $\left(m_{\kappa}\right)$ and on geophysical studies data $\left(m_{2}\right)$

превалирующее влияние на формирование добывных возможностей залежей.

3. Создана методика разделения эксплуатационных объектов на месторождениях с карбонатными коллекторами Волго-Уральской нефтегазоносной провинции по продуктивности с использованием данных, определение которых достаточно надёжно проводится на стадии окончания геологоразведочных мероприятий.

11. Innovative characterization of tight sandstones from Paleozoic basins in Poland using X-ray computed tomography supported by nuclear magnetic resonance and mercury porosimetry / P. Krakowskaa, E. Puskarczyka, M. Jędrychowskib, M. Habrata, P. Madejskic, M. Dohnalikd // Journal of Petroleum Science and Engineering. - 2018. - V. 166. - P. 389-405. DOI: 10.1016/j.petrol.2018.03.052.

12. Sander R., Pan Z., Connell L.D. Laboratory measurement of low permeability unconventional gas reservoir rocks: a review of experimental methods // Journal of Natural Gas Science and Engineering. - 2016. - V. 37. - P. 248-279. DOI: 10.1016/j.jngse.2016.11.041

13. Alvarado V., Thyne G., Murrell G.R. Screening strategy for chemical enhanced oil recovery in Wyoming basin // SPE Annual Technical Conference and Exhibition. - Denver, Colorado, USA, 21-24 September 2008. - 14 p. DOI: 10.2118/115940-MS.

14. Yakupov R.F., Mukhametshin V.Sh., Tyncherov K.T. Filtration model of oil coning in a bottom water-drive reservoir // Periodico Tche Quimica. - 2018. - V. 15. - Iss. 30. - P. 725-733.

15. Мухаметшин В.В., Андреев В.Е. Повышение эффективности оценки результативности технологий, направленных на расширение использования ресурсной базы месторождений с трудноизвлекаемыми запасами // Известия Томского политехнического университета. Инжиниринг георесурсов. - 2018. T. 329. - № 8. - С. 30-36

16. Дмитриевский А.Н. Ресурсно-инновационная стратегия развития экономики России // Нефтяное хозяйство. - 2017. № 5. - С. 6-7.

17. Геологические и технологические особенности создания гибких типовых шаблонов геологического моделирования / К.Е. Закревский, В.Л. Попов, А.Е. Лепилин, Е.А. Рыжиков // Нефтяное хозяйство. - 2020. - № 11. - С. 38-43. DOI: 10.24887/0028-2448-2020-11-38-43.

18. Мухаметшин В.В., Кулешова Л.С. О снижении уровня неопределенности при управлении заводнением залежей с трудноизвлекаемыми запасами // Известия Томского политехнического университета. Инжиниринг георесурсов. - 2020. T. 331. - № 5. - C. 140-146. DOI 10.18799/24131830/2020/5/2644. 
19. Use of hydrochloric acid to remove filter-cake damage from preformed particle gel during conformance-control treatments $/$ A. Imqam, B. Bai, M. Wei, H. Elue, F.A. Muhammed // SPE Production \& Operations. - 2016. - V. 31. - № 3. - 11 p. DOI: 10.2118/172352-PA.

20. Системное решение технологических проблем заканчивания строительства скважин / В.Н. Поляков, Ю.В. Зейгман, Ю.А. Котенёв, В.В. Мухаметшин, Ш.Х. Султанов, А.П. Чижов // Нанотехнологии в строительстве. - 2018. - Т. 10. - № 1. C. 72-87. DOI: $10.15828 / 2075-8545-2018-10-1-72-87$.

21. Рогачев М.К., Мухаметшин В.В. Контроль и регулирование процесса солянокислотного воздействия на призабойную зону скважин по геолого-промысловым данным // Записки Горного института. - 2018. - T. 231. - C. 275-280. DOI 10.25515/PMI.2018.3.275.

22. Ахметов Р.Т., Андреев А.В., Мухаметшин В.В. Методика прогноза остаточной нефтенасыщенности и коэффициента вытеснения по данным геофизических исследований для оценки эффективности применения нанотехнологий // Нанотехнологии в строительстве. - 2017. - Т. 9. - № 5. - С. 116-133. DOI: $10.15828 / 2075-8545-2017-9-5-116-133$
23. Kuleshova L.S., Mukhametshin V.V., Safiullina A.R. Applying information technologies in identifying the features of deposit identification under conditions of different oil-and gas provinces // Journal of Physics: Conference Series (ITBI 2019 - International Conference «Information Technologies in Business and Industry»). - 2019. - V. 1333. - Iss. 7 (072012). - P. 1-5. DOI: $10.1088 / 1742-6596 / 1333 / 7 / 072012$.

24. Идентификация слабо выработанных зон на месторождениях с трудноизвлекаемыми запасами / И.Ф. Хатмуллин, Е.И. Хатмуллина, А.Т. Хамитов, Р.А. Гималетдинов, С.Е. Мезиков // Нефтяное хозяйство. - 2015. - № 1. - С. 74-79.

25. Шустеф И.Н. О зависимости нефтеотдачи от продуктивности и гидропроводности пластов // РНГС Нефтегазовая геология и геофизика. - 1976. - № 8. - С. 15-16.

26. Шустеф И.Н. Геологические основы технологических решений в разработке нефтяных месторождений. - М.: Недра, 1988. -199 c

Поступила 28.10.2021 2.

\section{Информация об авторах}

Мухаметиин В.Ш., доктор геолого-минералогических наук, профессор, заведующий кафедрой разведки и разработки нефтяных и газовых месторождений, директор, филиал Уфимского государственного нефтяного технического университета в г. Октябрьском.

Кулешова Л.С., кандидат технических наук, доцент, преподаватель кафедры разведки и разработки нефтяных и газовых месторождений, заместитель директора по УР, филиал Уфимского государственного нефтяного технического университета в г. Октябрьском.

Cафиуллина $\boldsymbol{A . P . , ~ т е х н и к ~ к а ф е д р ы ~ р а з в е д к и ~ и ~ р а з р а б о т к и ~ н е ф т я н ы х ~ и ~ г а з о в ы х ~ м е с т о р о ж д е н и и ̆ , ~ ф и л и а л ~ У ф и м - ~}$ ского государственного нефтяного технического университета в г. Октябрьском. 
UDC 622.276 .63

\title{
GROUPING AND DETERMINING OIL RESERVOIRS IN CARBONATE RESERVOIRS BY THEIR PRODUCTIVITY AT THE STAGE OF GEOLOGICAL EXPLORATION
}

\author{
Vyacheslav Sh. Mukhametshin ${ }^{1}$, \\ vsh@of.ugntu.ru \\ Lyubov S. Kuleshova ${ }^{1}$, \\ mark212@mail.ru \\ Albina R. Safiullina', \\ safiullina.a.r@yandex.ru
1 Ufa State Petroleum Technological University, Branch of the University in the City of Oktyabrsky, 54a, Devonskaya street, Oktyabrsky, 452607, Russia.

\begin{abstract}
The relevance of the study is caused by the need to assess the productivity of fields in the Volga-Ural oil and gas province which are composed of a carbonate reservoir at the stage of project documents compiling with the aim of determining the profitability and development strategy of these facilities, as significant oil reserves are concentrated there and they are classified as hard-to-recover. Solving this problem allows us to expand the resource base of the region and minimize risks in making management decisions.

The purpose of the research is to group objects in carbonate reservoirs with the creation of a methodology for classifying objects going out from exploration into the category of high or low productivity applying indirect data.

Objects: oil deposits in carbonate reservoirs of the Volga-Ural oil and gas province.

Methods. The method of geological field analysis, some types of factor analysis were used when grouping.

Results. It was found that the boundary value of the oil deposits productivity index in carbonate reservoirs, referring the fields into highand low-productivity, is a value equal to $10 \mathrm{t} /$ day/MPa. It is shown that the separation of deposits by productivity is determined by the reservoir-filtration and thickness properties of the reservoir rocks, as well as the bedding conditions and the reservoir fluids properties. On average, these parameters are higher for highly productive facilities. Low-productivity objects, on average, have high reservoir oil viscosity and density values. It is impossible to clearly match the boundary value of the productivity coefficient to the boundary value of any parameter, since the productivity of the deposits is characterized by a complex of values of the reservoir parameters and the fluids saturating it, and only a set of these values makes it possible to evaluate it. A method was created for differentiating development facilities in Volga-Ural oil and gas province carbonate reservoirs by productivity index basing on indirect data to solve the problems of identifying production facilities in multi-layer fields; comparison of the facilities while conducting the development analysis, etc.
\end{abstract}

\section{Key words:}

Productivity index, oil, carbonate reservoirs, development efficiency, geological and physical properties of rocks.

\section{REFERENCES}

1. Muslimov R.Kh. Sovremennye metody povysheniya nefteizvlecheniya: proektirovanie, optimizatsiya $i$ otsenka effektivnosti [Modern methods of oil recovery increasing: design, optimization and performance evaluation]. Kazan, FEN Publ., 2005. $688 \mathrm{p}$.

2. Economides J.M., Nolte K.I. Reservoir stimulation. West Sussex, England, John Wiley and Sons, 2000. 856 p.

3. Mukhametshin V.V., Kuleshova L.S. Justification of lowproductive oil deposits flooding systems in the conditions of limited information amount. SOCAR Proceedings, 2019, no. 2 pp. 16-22. DOI: 10.5510/OGP20190200384. In Rus.

4. Byrnes A.P., Bhattacharya S. Influence of initial and residual oil saturation and relative permeability on recovery from transition zone reservoirs in shallow-shelf carbonates. SPE/DOE Symposium on Improved Oil Recovery. Tulsa, Oklahoma, USA, April 22-26, 2006. 11 p. DOI: $10.2118 / 99736-M S$.

5. Mukhametshin V.V. Efficiency estimation of nanotechnologies applied in constructed wells to accelerate field development. Nanotechnologies in Construction, 2018, vol. 10, no. 1, pp. 113 131. In Rus. DOI: 10.15828/2075-8545-2018-10-1-113-131.

6. Mukhametshin V.V. Oil production facilities management improving using the analogy method. SOCAR Proceedings, 2020 no. 4, pp. 42-50. DOI: 10.5510/OGP20200400464. In Rus.

7. Back M.J., Kirk G. An integrated portfolio management approach for more effective business planning. SPE Hydrocarbon Economics and Evaluation Symposium. Calgary, Alberta, Canada, September 24-25, 2012. 12 p. DOI: 10.2118/162748-MS.

8. Yakupov R.F., Mukhametshin V.Sh., Khakimzyanov I.N., Trofimov V.E. Optimization of reserve production from water oil zones of D3ps horizon of Shkapovsky oil field by means of horizontal wells. Georesursy, 2019, vol. 21, no. 3, pp. 55-61. DOI: 10.18599/grs.2019.3.55-61.

9. Burchette T. Carbonate rocks and petroleum reservoirs: a geological perspective from the industry. Geological Society of London Special Publications, 2012, vol. 370, pp. 17-37.

10. Roehl P.O., Choquette P.W. Carbonate petroleum reservoirs. New York, Springer-Verlag, 1985. $622 \mathrm{p}$.

11. Krakowskaa P., Puskarczyka E., Jędrychowskib M., Habrata M., Madejskic P., Dohnalikd M. Innovative characterization of tight sandstones from Paleozoic basins in Poland using X-ray computed tomography supported by nuclear magnetic resonance and mercury porosimetry. Journal of Petroleum Science and Engineering, 2018, vol. 166, pp. 389-405. DOI: 10.1016/j.petrol.2018.03.052

12. Sander R., Pan Z., Connell L.D. Laboratory measurement of low permeability unconventional gas reservoir rocks: a review of experimental methods. Journal of Natural Gas Science and Engineering, 2016, vol. 37, pp. 248-279. DOI: 10.1016/j.jngse.2016.11.041.

13. Alvarado V., Thyne G., Murrell G.R. Screening strategy for chemical enhanced oil recovery in Wyoming basin. SPE Annual Technical Conference and Exhibition. Denver, Colorado, USA, September 21-24, 2008. 14 p. DOI: 10.2118/115940-MS

14. Yakupov R.F., Mukhametshin V.Sh., Tyncherov K.T. Filtration model of oil coning in a bottom water-drive reservoir. Periodico Tche Quimica, 2018, vol. 15, Iss. 30, pp. 725-733.

15. Mukhametshin V.V., Andreev V.E. Increasing the efficiency of assessing the performance of techniques aimed at expanding the use of resource potential of oilfields with hard-to-recover reserves. Bulletin of the Tomsk Polytechnic University. Geo Assets Engineering, 2018, vol. 329, no. 8, pp. 30-36. In Rus. 
16. Dmitrievsky A.N. Resource-innovative strategy for the development of the Russian economy. Neftyanoe khozyaystvo $=$ Oil Industry, 2017, no. 5, pp. 6-7. In Rus.

17. Zakrevskiy K.E., Popov V.L., Lepilin A.E., Ryzhikov E.A Geological and technological features of creating flexible typical templates for geological modeling. Neftyanoe khozyaystvo $=$ Oil Industry, 2020, no. 11, pp. 38-43. In Rus. DOI: 10.24887/00282448-2020-11-38-43

18. Mukhametshin V.V., Kuleshova L.S. On uncertainty level reduction in managing waterflooding of the deposits with hard to extract reserves. Bulletin of the Tomsk Polytechnic University. Geo Assets Engineering, 2020, vol. 331, no. 5, pp. 140-146. In Rus. DOI: $10.18799 / 24131830 / 2020 / 5 / 2644$

19. Imqam A., Bai B., Wei M., Elue H., Muhammed F.A. Use of hydrochloric acid to remove filter-cake damage from preformed particle gel during conformance-control treatments. SPE Production \& Operations, 2016, vol. 31, no. 3, 11 p. DOI: 10.2118/172352-PA.

20. Polyakov V.N., Zeigman Yu.V., Kotenev Yu.A., Mukhametshin V.V., Sultanov Sh.Kh., Chizhov A.P. System solution for technological problems of well construction completion. Nanotechnologies in Construction, 2018, vol. 10, no. 1, pp. 72-87. In Rus. DOI 10.15828/2075-8545-2018-10-1-72-87.

21. Rogachev M.K., Mukhametshin V.V. Control and regulation of the hydrochloric acid treatment of the bottomhole zone based on field-geological data. Journal of Mining Institute, 2018, vol. 231, pp. 275-280. DOI: 10.25515/PMI.2018.3.275.
22. Akhmetov R.T., Andreev A.V., Mukhametshin V.V. Residual oil saturation and the displacement factor prediction methodology based on geophysical studies data to evaluate efficiency of nanotechnologies application. Nanotechnologies in Construction, 2017, vol. 9, no. 5, pp. 116-133. In Rus. DOI: 10.15828/20758545-2017-9-5-116-133.

23. Kuleshova L.S., Mukhametshin V.V., Safiullina A.R. Applying information technologies in identifying the features of deposit identification under conditions of different oil-and gas provinces. Journal of Physics: Conference Series (ITBI 2019 - International Conference «Information Technologies in Business and Industry»), 2019, vol. 1333, Iss. 7 (072012), pp. 1-5. DOI: 10.1088/1742-6596/1333/7/072012.

24. Khatmullin I.F., Khatmullina E.I., Khamitov A.T., Gimaletdinov R.A., Mezikov S.E. Identification of zones with poor displacement in fields with hard-to-recover reserves. Neftyanoe khozyaystvo $=$ Oil Industry, 2015, no. 1, pp. 74-79. In Rus.

25. Shustef I.N. O zavisimosti nefteotdachi ot produktivnosti i gidroprovodnosti plastov [On the dependence of oil recovery on the productivity and hydroconductivity of layers]. RNGS Neftegazovaya geologiya i geofizika, 1976, no. 8, pp. 15-16.

26. Shustef I.N. Geologicheskie osnovy tekhnologicheskikh resheniy v razrabotke neftyanykh mestorozhdeniy [Geological bases of technological solutions in the development of oil fields]. Moscow, Nedra Publ., 1988. 199 p.

Received: 28 October 2021.

\section{Information about the authors}

Vyacheslav Sh. Mukhametshin, Dr. Sc., professor, head of the Department of Oil and Gas Field Exploration and Development, director, Ufa State Petroleum Technological University, Branch of the University in the City of Oktyabrsky.

Lyubov S. Kuleshova, Cand. Sc., assotiate professor, deputy director for Academic Affairs, Ufa State Petroleum Technological University, Branch of the University in the City of Oktyabrsky.

Albina R. Safiullina, technician, Ufa State Petroleum Technological University, Branch of the University in the City of Oktyabrsky. 